\title{
Simplified modeling of EM field coupling to complex cable bundles
}

\author{
B. Schetelig ${ }^{1}$, J. Keghie ${ }^{1}$, R. Kanyou Nana ${ }^{1}$, L.-O. Fichte ${ }^{1}$, S. Potthast ${ }^{2}$, and S. Dickmann ${ }^{1}$ \\ ${ }^{1}$ Faculty of Electrical Engineering, Helmut-Schmidt-University/University of the Federal Armed Forces Hamburg, Germany \\ ${ }^{2}$ Bundeswehr Research Institute for Protective Technologies and NBC Protection (WIS), Munster, Germany
}

\begin{abstract}
In this contribution, the procedure "Equivalent Cable Bundle Method" is used for the simplification of large cable bundles, and it is extended to the application on differential signal lines. The main focus is on the reduction of twisted-pair cables. Furthermore, the process presented here allows to take into account cables with wires that are situated quite close to each other. The procedure is based on a new approach to calculate the geometry of the simplified cable and uses the fact that the line parameters do not uniquely correspond to a certain geometry. For this reason, an optimization algorithm is applied.
\end{abstract}

\section{Introduction}

To assure the immunity of cable-wired communication systems to external electromagnetic influences, one needs to know the disturbances coupled into the connecting cables to carry out an evaluation at the inputs of the connected devices based on its prescriptive limits. From several research projects, it is known that coupling via the cables is often quite important because of their extended geometry, compared to the direct coupling via the enclosures. Especially on ships, we have to deal with quite extensive cable bundles. Numerical calculations of such cable structures place great demands on computation power. An analytical approach is mostly restricted to quite simple geometries. Within this context, a methodology can be applied that can be found in literature (Andrieu, 2006; Andrieu et al., 2008). This procedure is based on the idea to combine these wires of a cable harness that show similar behaviour while being irradiated by an electromagnetic field. In this way, the number of wires to be considered can be reduced. The behaviour of this simplified cable bundle to electromagnetic irradiation remains

Correspondence to: B. Schetelig (benedikt.schetelig@hsu-hh.de) unchanged when compared to the original one. Especially when very extensive cable bundles are analyzed, there is the opportunity of a considerably large reduction of the bundle geometry.

After applying this methodology to a cable bundle, its immunity to EMI can be calculated later on by using any wellestablished tools and procedures. The reduced cable bundle can be treated the same way as any other harness. Any kind of calculation method can be applied, but the application of the reduction technique is particularly appropriate when using numerical methods such as the method of moments. When we apply numerical procedures on the simplified harness we get a significant reduction of the necessary number of mesh cells or segments when compared to the original, much larger cable bundle. This means reduced demands on computation power. Analytical approaches are simplified, too.

The "Equivalent Cable Bundle Method" was formerly used for the reduction of multiconductor transmission lines with a reference conductor that is formed by a common ground plane. The resulting voltages and currents therefore are common mode values referred to the ground plane. In a scenario close to reality, particularly the analysis of differential signal lines matters. Within this context, this paper presents a modification of the reduction technique to allow its application to differential signal lines, too. Nevertheless, the obtained insight can likewise be applied to the calculation of disturbances on cables with reference to ground.

\section{Fundamentals of transmission line theory}

The methodology of simplified modelling of field coupled disturbances is based on the transmission line analysis of the cable bundles of interest. The use of transmission line theory to describe the coupling of electromagnetic fields is wellknown and was described by (Tesche et al., 1997; Paul, 1994)

Published by Copernicus Publications on behalf of the URSI Landesausschuss in der Bundesrepublik Deutschland e.V. 


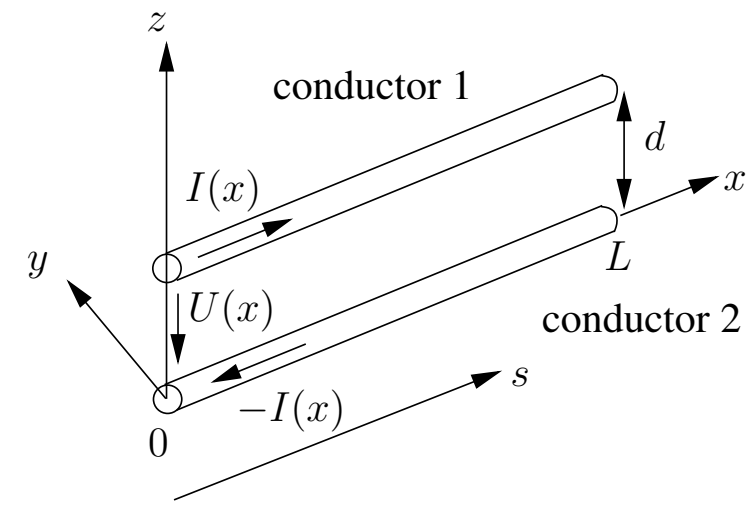

Fig. 1. Geometrical adjustment of the transmission line.

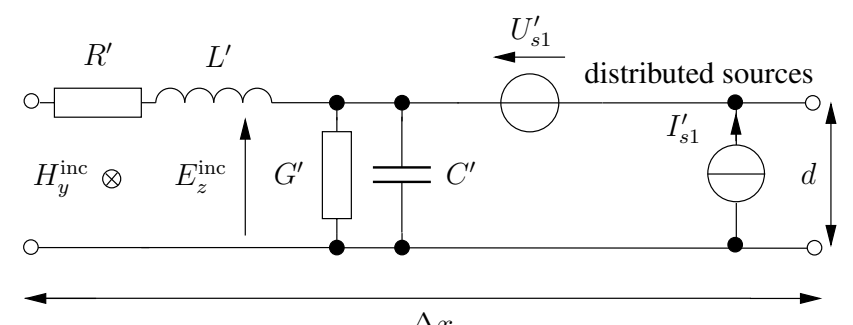

Fig. 2. Equivalent circuit of an irradiated single conductor transmission line by Taylor approach.

among others. In the following, we want to summarize the basic facts, that are used in the context of this study.

The behaviour of voltages and currents on transmission lines can be described by the telegrapher's equations (cf. Figs. 1, 2):

$$
\left\{\begin{array}{l}
-\frac{\mathrm{d} U}{\mathrm{~d} x}=\left(R^{\prime}+\mathrm{j} \omega L^{\prime}\right) \cdot I, \\
-\frac{\mathrm{d} I}{\mathrm{~d} x}=\left(G^{\prime}+\mathrm{j} \omega C^{\prime}\right) \cdot U .
\end{array}\right.
$$

$R^{\prime}, L^{\prime}, G^{\prime}$ and $C^{\prime}$ are the per-unit-length line parameters.

If we assume a lossless transmission line and therefore neglect $R^{\prime}$ and $G^{\prime}$, the characteristic impedance then can be noted as follows:

$Z_{0}=\sqrt{\frac{L^{\prime}}{C^{\prime}}}$.

The coupling of external electromagnetic fields to transmission lines was described by Taylor as well as by Agrawal and Rachidi by using different approaches. As summarized by (Tesche, 1995), the influence of the electromagnetic field can be represented by additional sources in the equivalent circuit diagramm of the transmission line. In the general formulation by Taylor, voltage sources as well as current sources

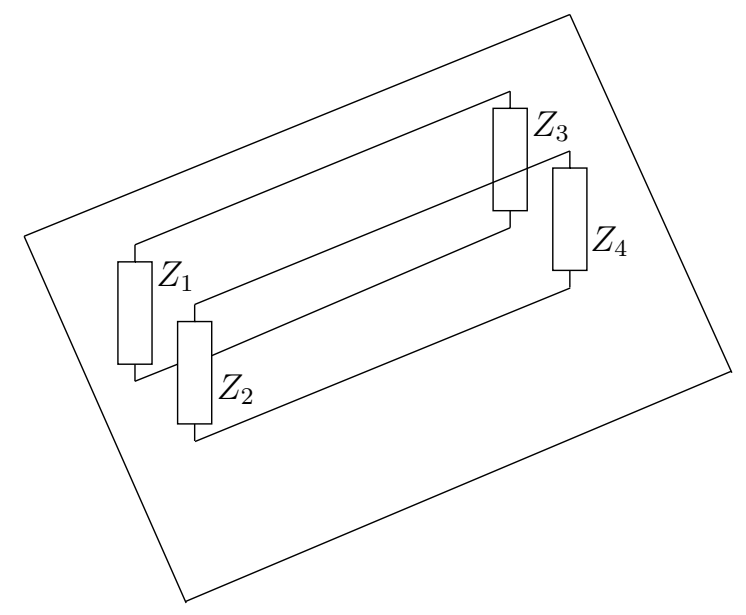

Fig. 3. Transmission lines with differential termination loads.

are used. The telegrapher's equations (Eq.1) are modified as follows:

$\left\{\begin{array}{l}\frac{\mathrm{d} U}{d x}+\mathrm{j} \omega L^{\prime} \cdot I=U_{s 1}^{\prime}, \\ \frac{\mathrm{d} I}{d x}+\mathrm{j} \omega C^{\prime} \cdot U=I_{s 1}^{\prime} .\end{array}\right.$

The distributed sources can be described as

$$
\begin{aligned}
U_{s 1}^{\prime} & =-\mathrm{j} \omega \mu_{0} \int_{0}^{d} H_{y}^{\mathrm{inc}} \mathrm{d} z, \\
I_{s 1}^{\prime} & =-\mathrm{j} \omega C^{\prime} \int_{0}^{d} E_{z}^{\mathrm{inc}} \mathrm{d} z,
\end{aligned}
$$

with reference to Fig. 2.

\section{Methodological approach}

The simplification of cable bundles using differential signal lines is similar to the methodology for signal lines referred to the ground plane. This procedure was presented in detail in (Andrieu et al., 2008).

The scenario of the modified methodology is presented in Figs. 3 and 4: We analyze a cable bundle with a constant distance to the ground. The wires are surrounded by a homogeneous dielectric. The attached devices are represented by their input impedances. As we focus on differential signal transmission, no common mode terminations exist.

The first step of reducing the profile of the bundle is done by assuming a similar coupling of different wires. For these wires we assume a similar current distribution in the lines, so in the following we can categorize all the wires to individual groups with similar attributes. In the sample setup in Fig. 4, there are two differential signal lines consisting of the wires 


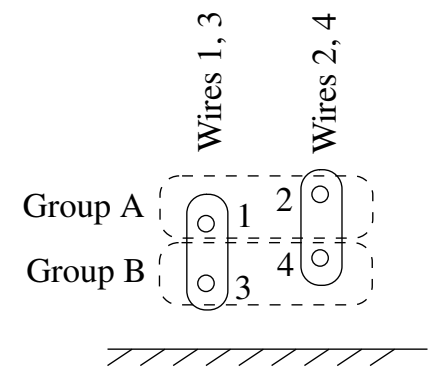

equiv. cable A

equiv. cable $\mathrm{B}$

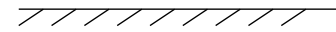

Fig. 4. Cross section of the original (left) and reduced (right) cable of Fig. 3 (principle diagram with two pairs).

1,3 and the wires 2, 4 respectively. We assume that the wires 1,2 have a similar coupling behaviour and label them group A. Group B consists of the wires 3, 4. If we focus on differential lines, we have to take into account the restriction that it is not useful to allocate both wires of the same wire pair to the same group.

Now, a general rule how to group the wires has to be developed. As already mentioned, the basic arrangement criterion is, that all wires which are placed in one group have to behave the same way (or very similar) in terms of the field to transmission line coupling. The susceptibility strongly depends on the relation of the characteristic impedance and the termination loads. This is why in the referred procedure developed by (Andrieu et al., 2008) the coupling of the wires is characterized by the relation of the common mode terminations to the common mode characteristic impedance $Z_{\mathrm{cm}}$. This rule allows up to four groups: In the first group, both common mode terminations are smaller than the common mode characteristic impedance. In the second group, both terminations are larger than the common mode characteristic impedance. In the third group, e.g. the termination at the left end is smaller and the one at the right end is larger than $Z_{\mathrm{cm}}$. In the fourth group, the size of the terminations is alternated. Following this rule, a differential signal cable with several pairs and without common mode terminations is reduced to a single pair of wires. In applications where identical wires with identical terminations are used (e.g. LAN or any other bus system), we always get a reduction to two groups. As mentioned before, it is not useful to place both wires of a differential pair in one group, even if their susceptibility is very similar.

\section{Line parameters of the reduced cable bundle}

Now, all the wires in each of these groups have a similar coupling behaviour. In the following we want to reduce each of these groups to single equivalent wires that show the same coupling behaviour as the sum of the single wires of each group. This can be done by making the following assumptions.
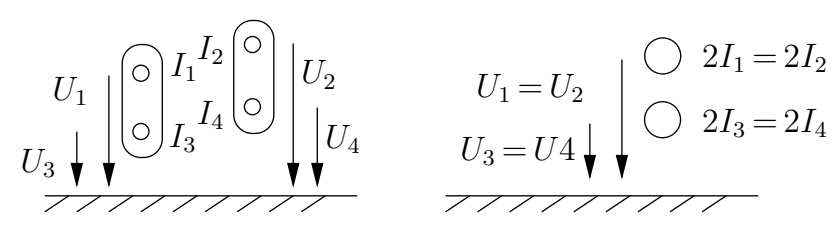

Fig. 5. Voltages and currents of the original (left) and reduced (right) cable.

1. The induced voltages in each of the wires of one group are the same.

2. All currents in the different wires of one group are equal.

If we compare the original cable bundle with the reduced one (cf. Fig. 4), the following observations can be derived (Fig. 5):

1. The induced voltages in each of the wires of one group of the original cable and the one of the belonging equivalent conductor of the simplified cable are the same.

2. The sum of all currents in all conductors of a group equals the current in the equivalent simplified conductor.

Applying these rules, Eq. (6), which represents the telegrapher's equations for a four-wire cable bundle (cf. Figs. 4, 5), can be simplified:

$$
\left\{\begin{aligned}
\frac{\mathrm{d}}{\mathrm{d} x}\left(\begin{array}{l}
I_{1} \\
I_{2} \\
I_{3} \\
I_{4}
\end{array}\right) & =-\mathrm{j} \omega\left(\begin{array}{llll}
C_{11} & C_{12} & C_{13} & C_{14} \\
C_{21} & C_{22} & C_{23} & C_{24} \\
C_{31} & C_{32} & C_{33} & C_{34} \\
C_{41} & C_{42} & C_{43} & C_{44}
\end{array}\right)^{\prime}\left(\begin{array}{l}
U_{1} \\
U_{2} \\
U_{3} \\
U_{4}
\end{array}\right), \\
\frac{\mathrm{d}}{\mathrm{d} x}\left(\begin{array}{l}
U_{1} \\
U_{2} \\
U_{3} \\
U_{4}
\end{array}\right) & =-\mathrm{j} \omega\left(\begin{array}{llll}
L_{11} & L_{12} & L_{13} & L_{14} \\
L_{21} & L_{22} & L_{23} & L_{24} \\
L_{31} & L_{32} & L_{33} & L_{34} \\
L_{41} & L_{42} & L_{43} & L_{44}
\end{array}\right)^{\prime}\left(\begin{array}{l}
I_{1} \\
I_{2} \\
I_{3} \\
I_{4}
\end{array}\right) .
\end{aligned}\right.
$$

Assuming that group A consists of the wires 1 and 2 and that group B consists of the wires 3 and 4, these equations can be written the following way by applying simple matrix operations:

$$
\left\{\begin{array}{l}
\frac{\mathrm{d}}{\mathrm{d} x}\left(\begin{array}{l}
2 I_{1} \\
2 I_{3}
\end{array}\right)=-\mathrm{j} \omega \cdot \mathbf{C}_{\text {red }}^{\prime} \cdot\left(\begin{array}{l}
U_{1} \\
U_{3}
\end{array}\right), \\
\frac{\mathrm{d}}{\mathrm{d} x}\left(\begin{array}{l}
U_{1} \\
U_{3}
\end{array}\right)=-\mathrm{j} \omega \cdot \mathbf{L}_{\text {red }}^{\prime} \cdot\left(\begin{array}{l}
2 I_{1} \\
2 I_{3}
\end{array}\right),
\end{array}\right.
$$

with: 

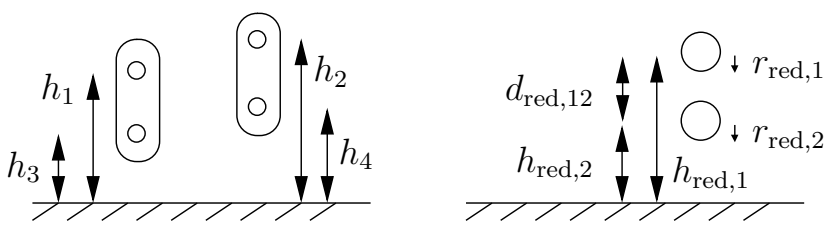

Fig. 6. Geometry of the original (left) and reduced (right) cable.

$\mathbf{C}_{\text {red }}^{\prime}=\left(\begin{array}{ll}C_{11}+C_{12}+C_{21}+C_{22} & C_{13}+C_{14}+C_{23}+C_{24} \\ C_{31}+C_{32}+C_{41}+C_{42} & C_{33}+C_{34}+C_{43}+C_{44}\end{array}\right)^{\prime}$,

$\mathbf{L}_{\text {red }}^{\prime}=\left(\frac{\frac{L_{11}+L_{12}+L_{21}+L_{22}}{4} \frac{L_{13}+L_{14}+L_{23}+L_{24}}{4}}{\frac{L_{31}+L_{32}+L_{41}+L_{42}}{4} \frac{L_{33}+L_{34}+L_{43}+L_{44}}{4}}\right)^{\prime}$.

This yields a reduced cable bundle with only two equivalent wires with a reduced capacitance matrix $\mathbf{C}_{\text {red }}^{\prime}$ and a reduced inductance matrix $\mathbf{L}_{\text {red. }}^{\prime}$.

\section{Termination loads}

If we focus on differential signals, we consider only differential termination loads, too. Arranging the wires of the different pairs to various groups, the differential terminations are set in parallel. So, the resulting terminations of the equivalent pairs equal the parallel connections of the belonging original terminations.

\section{Geometry of the reduced cable bundle}

Following the procedure presented in the previous chapter, we get the system of differential equations (Eq. 7) that characterizes the reduced cable bundle. If we want to use field simulation tools on the reduced cable bundle we need to extract the resulting geometry of the reduced cable bundle from the determined p.u.l. (per-unit-length) line parameters. This can be done by the following procedure. It was developed for symmetric signal lines, but can also applied to asymmetric ones.

\subsection{Determination of estimates}

The heights above ground of the equivalent conductors can be estimated by taking the average of the heights of all the conductors of the belonging group (cf. Fig. 6):

$h_{\mathrm{red}, i}=\frac{1}{n} \sum_{k=1}^{n} h_{k}$,

with $n$ : number of wires in group $i$.
The radii of the equivalent wires can be determined by the conversion of the analytical formula of the self inductance $L_{\text {red, }, i}^{\prime}$ :

$L_{\mathrm{red}, i i}^{\prime}=\frac{\mu}{2 \pi} \ln \frac{2 h_{\mathrm{red}, i}-r_{\mathrm{red}, i}}{r_{\mathrm{red}, i}}$.

The equation for the mutual inductance (concerning the conductors $i, j$ ) can be used to determine the distance $d_{\text {red, } i j}$ between two equivalent conductors:

$L_{\mathrm{red}, i j}^{\prime}=\frac{\mu}{2 \pi} \ln \left(\frac{d_{\mathrm{red}, i j}-r_{\mathrm{red}, j}}{s_{2}-r_{\mathrm{red}, j}}\right)$,

with:

$s_{2}=\sqrt{\left(h_{\mathrm{red}, i}+h_{\mathrm{red}, j}\right)^{2}+d_{\mathrm{red}, i j}^{2}-\left(h_{\mathrm{red}, j}-h_{\mathrm{red}, i}\right)^{2}}$,

with $d_{\text {red, } i j}$ being the distance between conductor $i$ and conductor $j$. The distance between the mirror image of conductor $i$ (because of the metallic ground) and conductor $j$ is named $s_{2}$.

\subsection{Optimization of the geometry}

If we use the results of the geometry parameters as described in Sect. 6.1 for further susceptibility calculations of asymmetric signal lines (return conductor: ground), we get quite a good accordance of the sum of the induced currents of the original and the current of the reduced cable bundle. Applying this method to differential cables and particularly to twisted pairs, the accordance is getting very poor. Besides, the results of the first estimation usually are not coherent. Looking at them in detail, we find out that the distance $d_{\text {red }, i j}$, calculated from Eq. (10), and the distance which can be derived from the difference of the $h_{\mathrm{red}, i}, h_{\mathrm{red}, j}$ are not equal. This comparison can only be done if we use a geometry where the two wires of a pair are located above each other. This is a restriction if uniform lines are analyzed, but is no problem when stepping over to twisted pairs.

The contradiction of the incoherent geometry parameters can be solved by applying an optimization algorithm on the estimates. The estimated values, calculated above, individually match the corresponding inductance matrix perfectly well (where they were derived from). But the geometry of the simplified cable bundle, associated to the matrix, is not unambiguous and the geometry parameters of the first estimation do not necessarily match to each other. So, the task is, to find a solution where all parameters harmonize and all Eqs. $(8,9,10)$ are still fulfilled.

The capacitance matrix $\mathbf{C}^{\prime}$ red does not provide further information if a homogene dielectric is assumed, because $\mathbf{L}_{\text {red }}^{\prime}$ and $\mathbf{C}^{\prime}$ red are directly connected via the propagation velocity:

$\mathbf{C}_{\text {red }}^{\prime}=\frac{1}{v^{2}} \mathbf{L}_{\text {red }}^{\prime-1}$ 
We can state the following conditions, which have to be satisfied by the optimization:

1. The distances of the wires of the reduced cable bundle equal the differences of the first estimates of the heights of the reduced cable bundle, Eq. (8): $d_{\text {red }, i j}=$ $\left|h_{\text {red }, i}-h_{\text {red, } j}\right|$. That means that $d_{\text {red }, i j}$ remains unchanged during the optimization process. This starting condition guarantees, that the total diameter of the equivalent cable remains the same, compared to the original cable.

2. As $d_{\text {red }, i j}$ is fixed, the $h_{\text {red }, i}, h_{\text {red }, j}$ have to be modified to fulfill $d_{\text {red, } i j}=\left|h_{\text {red }, i}-h_{\text {red }, j}\right|$.

3. When the optimization process in finished, the final reduced geometry still has to correspond to the the line parameters of the reduced cable bundle (cf. Eqs. 8, 9, $10)$.

Using these conditions, the optimization procedure is structured as follows:

1. Estimate $h_{\mathrm{red}, i}, h_{\mathrm{red}, j}$ as the average of the original conductors of the corresponding group.

2. Set $d_{\mathrm{red}, i j}$ fix as defined in Eq. (1) according to the original geometry.

3. Recalculate the height $h_{\text {red, } i}$ of the lower wire $i$, using $d_{\text {red, }, i j}$ from Eq. (2) and $h_{\text {red, } j}$ by applying Eq. (10).

4. Now, the error can be calculated by comparing the difference of the heights with distance $d_{\text {red, } i j}$ Eq. (2).

5. If the error exceeds the predefined tolerance, the height $h_{\text {red, } j}$ of the upper wire $j$ is adjusted by adding (subtracting) a portion of the error, depending on the polarity of the calculated error

6. A next iterative loop is started by continuing with Eq. (3).

After some steps and according to the defined tolerance, the curve convergences, as to be seen in Fig. 7.

There are different ways of implementing the conditions mentioned above. They result in a different number of necessary steps to reach the convergence condition. It is very likely to get different results for the geometry of the reduced cable bundle with little differences in coupling behaviour. The variation presented here, is a very simple one, producing quite good results (see Sect. 8).

By applying this code, a second problem to be mentioned is solved, too. During the reduction of the cable bundle, the radii of the simplified cable tend to grow. This depends on the reduction of the $L_{i i}^{\prime}$ (cf. Eq. 12) when moving from Eq. (6) to Eq. (7). As a consequence it is not possible to apply the cable reduction technique if the original cables have very little distance, since the reduced cable would overlap. When

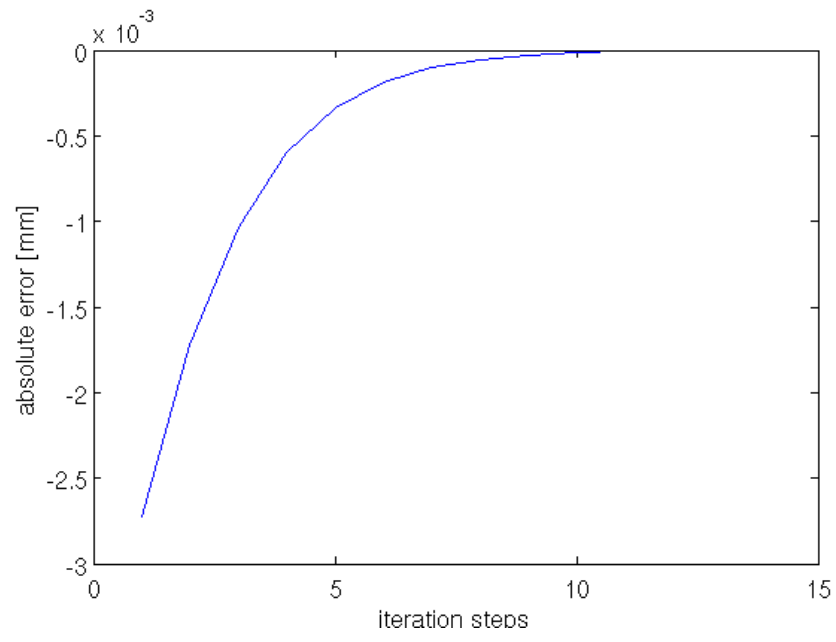

Fig. 7. Error reduction due to recursive iterations.

the geometry is varied recursively in the context of the optimization code, the heights are reduced, compared to the first estimate of the reduced geometry, and as Eq. (12) shows, the result is a reduction of the radii, too:

$r_{\mathrm{red}, i}=\frac{2 \cdot h_{\mathrm{red}, i}}{\exp \left(\frac{L_{\mathrm{red}, i i}^{\prime} \cdot 2 \pi}{\mu}\right)}$.

We know from experience that it is even possible to cut away the overlapping radii (without touching the distance) to about $70 \%$ causing quite little error.

\section{Twisted-pair cables}

The application of this procedure on twisted-pair cables seems to be very difficult at the beginning: The procedure described above depends on the line parameters of the original as well as the reduced cable. The determination of these parameters initially requires, by definition, a homogene geometry with a constant distance to the ground. That is not the case for twisted cables. There are several studies that have a close look at the problem how to apply transmission line theory to nonuniform cables. (Nitsch and Gronwald, 1999) uses a generalized telegrapher's equation. (Omid, 1997) adopts a method of an equivalent cascaded network chain. In the context of reduction of twisted-pair cables, a much more simple methodology can be applied and leads to satisfying results.

The approach is based on a chained transmission line analysis too. What we are looking for is the replacement of a large bundle of twisted pairs by a reduced bundle with only one twisted pair (Fig. 8). This is an enormous simplification of the nonuniform structure. 

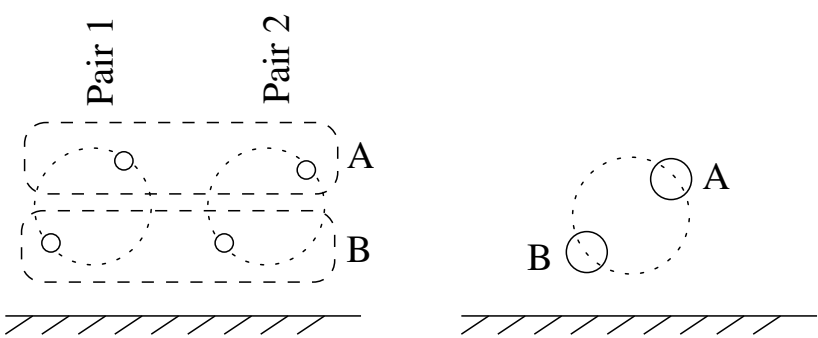

Fig. 8. Principle of reducing twisted-pair cables: Original cable bundle (left) and reduced cable (right).

To demonstrate this simplification approach, we want to analyze one twist of the original cable. The $360^{\circ}$ twist is divided exemplarily into four parts (angle of difference $\Delta \alpha=90^{\circ}$ ), as illustrated in Fig. 9. These parts can now be regarded separately. We reduce the twisted pairs in each part of the twist in the way as shown on the left side of Fig. 8 . The arrangement of the wires is done in the same way as it is done with uniform cables. When we now have a look at the geometry of the reduced system, we see that the reduction of twisted pairs yields a twisted pair again. This is because the wires we group together always have the same relation to each other in every position of the twist. This can been seen exemplarily in Fig. 9 for four steps. Of course, because of the change of height of each wire, the resulting radii of the reduced cable are not exactly the same. As the difference can be neglected in practical configurations, these radii can be averaged. Hence, it is possible to simplify cable bundles with several twisted pairs to reduced ones with only one pair. Additionally, it is possible to perform the total reduction process and the calculation of the reduced geometry only on the geometry of part 1 of Fig. 9. The results then can be used to model the reduced twisted pair in the CAD environment of the field simulation tool. In this way, it possible to treat twisted-pair cables during the reduction process in the same way as uniform cables, if the pitch length (incl. orientation) as well as the wire distances of the different original pairs are the same. If one of these criteria is violated, the resulting equivalent cable pair is no longer an ideal helix structure and averaging had to be applied.

\section{Validation}

To verify the presented method, a MATLAB script was implemented to cover the total reduction process starting with the original geometry and ending with the reduced geometry. The total procedure of reduction is structured as follows:

1. Analytic calculation of the line parameters according to the original geometry.

2. Generation of the line parameters of the reduced cable bundle from the original cable bundle.

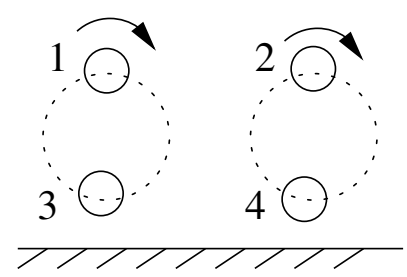

(a) Part $1\left(0^{\circ}\right)$

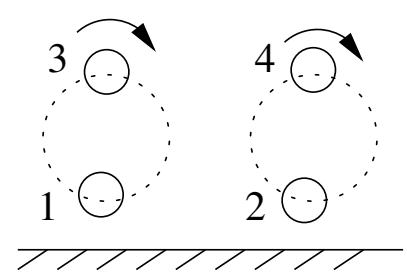

(c) Part $3\left(180^{\circ}\right)$

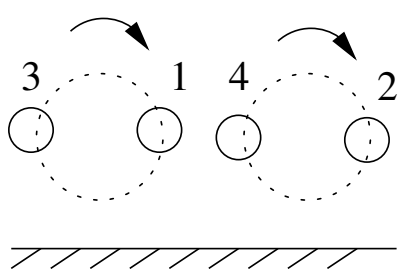

(b) Part $2\left(90^{\circ}\right)$

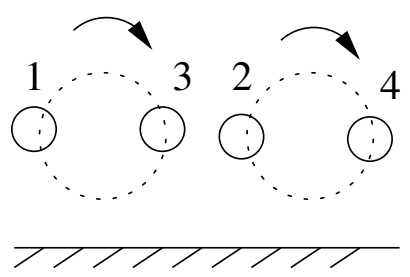

(d) Part $4\left(270^{\circ}\right)$
Fig. 9. Stepwise analysis of a $360^{\circ}$ twist of a twisted-pair cable.

3. First estimation of the geometry of the reduced cable by applying the same equations as in Eq. (1).

4. Application of the iterative optimization run.

5. Modelling the reduced geometry in a 3D field simulation program (FEKO) and comparing the induced currents to the currents from the simulation run of the original cable.

The basic geometrical parameters of the original cable used for the following validations are:

$\begin{array}{ll}\text { height of the lower wire } & 5 \mathrm{~mm} \\ \text { wire radius } & 0.1 \mathrm{~mm} \\ \text { distance between twisted wires } & 0.5 \mathrm{~mm} \\ \text { distance between two pairs } & 0.5 \mathrm{~mm} \\ \text { length of twist (for twisted pairs) } & 20 \mathrm{~mm}\end{array}$

The excitation of the transmission lines is a vertically polarized plane EM wave with a poynting vector perpendicular to the cable bundle.

First, we want to compare the results for differential pairs of uniform wires. In Fig. 10, the currents in the original and reduced cable bundles are plotted. Note the good accordance between the curves. The amplitudes as well as the resonance peaks match very well.

Figure 11 shows the comparison of the currents in the original and the reduced twisted harness. As we can see, the curves match quite well. There is only a little difference in the resonance peaks. This can be explained by the fact, that by reducing the cable, the propagation velocity is shifted a tiny amount.

$v^{2}=\mathbf{L}^{\prime-1} \cdot \mathbf{C}^{\prime-1}$ 


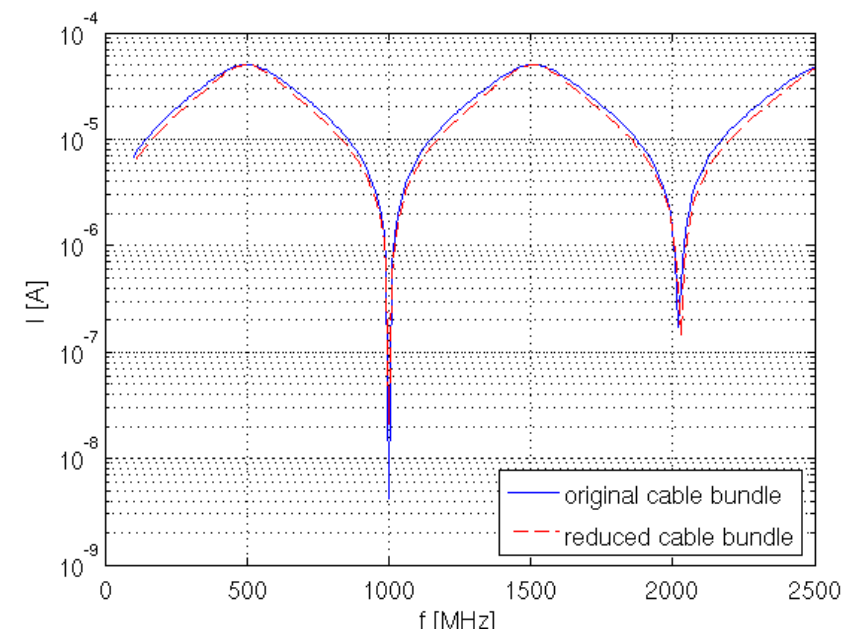

Fig. 10. Comparison of the induced current on a uniform, differential cable.

This is because the product of the p.u.l.-parameters $\mathbf{L}_{\text {orig }}^{\prime}$ and $\mathbf{C}^{\prime}$ orig of the original cable does not equal the product $\mathbf{L}_{\text {red }}^{\prime}$ and $\mathbf{C}_{\text {red }}^{\prime}$ of the reduced cable. One way to improve the accordance is to add a dielectric coating to the wires of the reduced cable bundle (Andrieu, 2006). This would affect the capacitance and in that way, the propagation velocity of the reduced cable could be adapted.

Finally, we want to review the improved possibility to apply the reduction method to cables with wires that are situated very close to each other. The advantage can be summarized as a reduced growth of the radii in the context of the simplification. The radii of the simplified cable in this example are $r_{\text {red,est }}=0.32 \mathrm{~mm}$ if the optimization algorithm is not applied. If the optimized geometry data is used, the radii are reduced to an average of $r_{\text {red,opt }}=0.21 \mathrm{~mm}$. This means a reduction to $66 \%$ of the original size and the arising space between the wire can be used for a narrower adjustment of the original cable bundle. The agreement between the original and the reduced cable bundles still remains good as shown in Figs. 10 and 11.

\section{Conclusions}

In this paper an approach was derived to extend the Equivalent Cable Bundle Method to differential cables. We presented an optimization algorithm that enabled the application on twisted-pair cables and the analysis of cable bundles with very little distance between the wires. As shown in the validation chapter, this methodology yields to a good accordance of the simplified cable bundle with the original one. Hence, the methodology allows to perform a simplified modelling of EM fields to differential transmission lines.

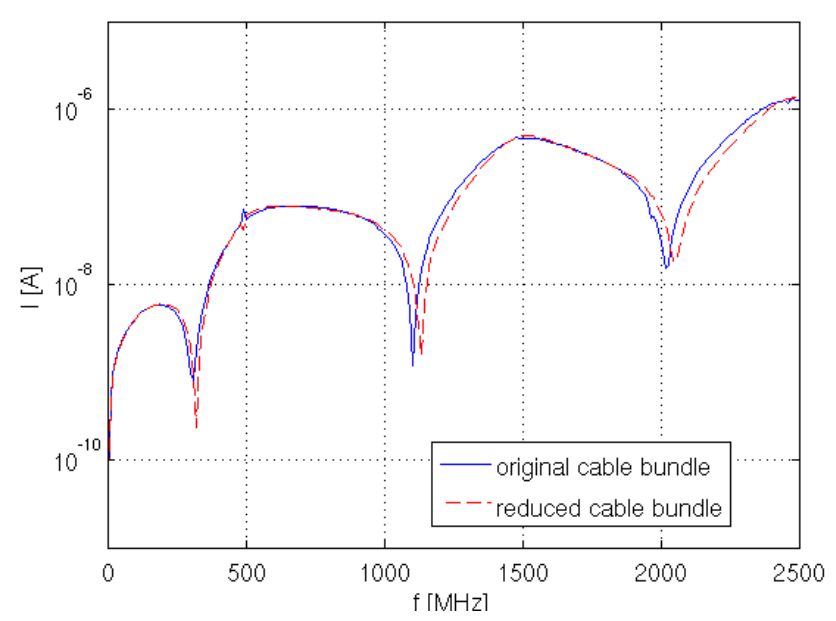

Fig. 11. Comparison of the induced current on a twisted-pair cable.

\section{References}

Andrieu, G.: Elaboration et application d'une méthode de faisceau équivalent pour l'étude des couplages électromagnetiques sur réseaux de câblages automobiles, Ph.D. thesis, Lille University, 2006.

Andrieu, G., Koné, L., Bocquet, F., Démoulin, B., and Parmantier, J.-P.: Multiconductor Reduction Technique for Modeling Common-Mode Currents on Cable Bundles at High Frequency for Automotive Applications, IEEE Transactions on electromagnetic compatibility, 50, 175-184, 2008.

Nitsch, J. and Gronwald, F.: Analytical Solutions in Nonuniform Multiconductor Transmission Line Theory, IEEE Transactions on electromagnetic compatibility, 41, 469-479, 1999.

Omid, M.: Field Coupling to Nonuniform and Uniform Transmission Lines, IEEE Transactions on electromagnetic compatibility, 39, 201-211, 1997.

Paul, C. R.: Analysis of Multiconductor Transmission Lines, Wiley, 1994.

Tesche, F. M.: Principles and applications of EM field coupling to transmission lines, EMC Zurich Symposium, 9, 21-31, 1995.

Tesche, F. M., Ianoz, M. V., and Karlsson, T.: EMC Analysis Methods and Computational Methods, Wiley, 1997. 